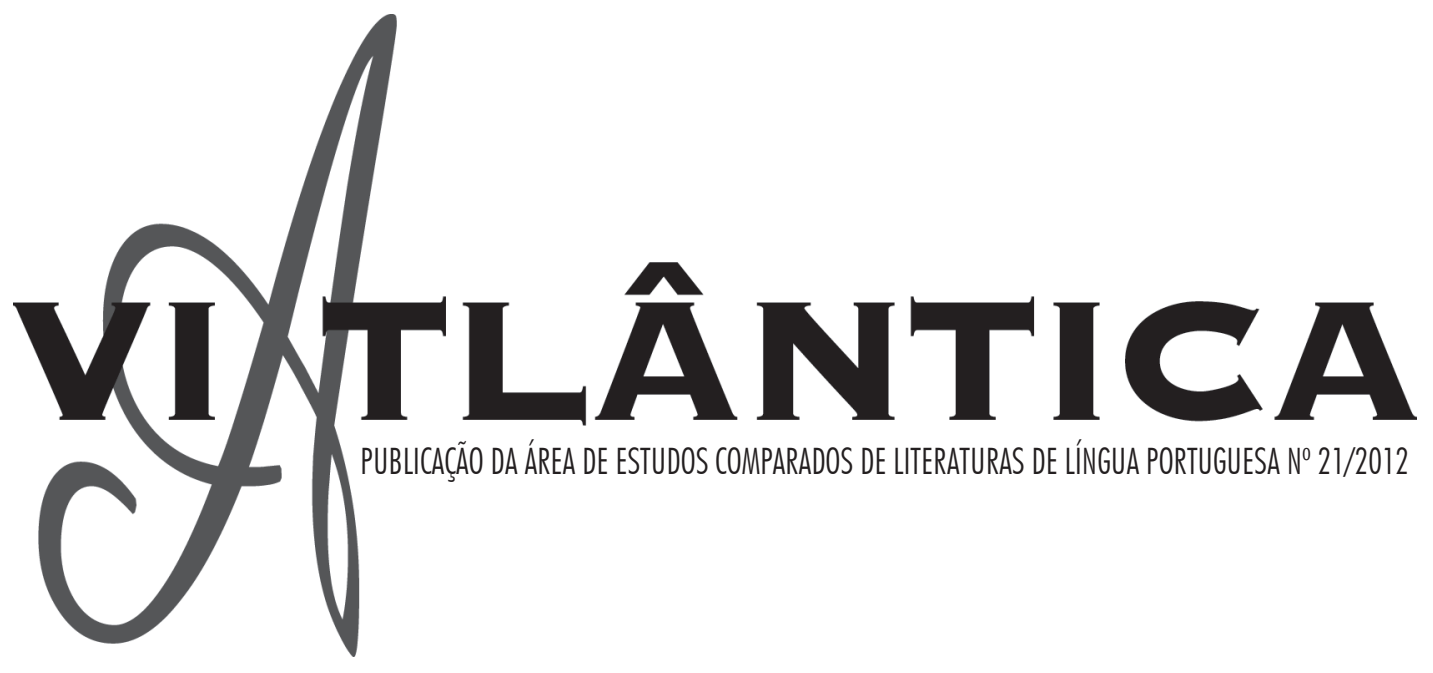


UNIVERSIDADE DE SÃO PAULO

Reitor: João Grandino Rodas

Vice-Reitor: Helio Nogueira da Cruz

FACULDADE DE FILOSOFIA, LETRAS E CIÊNCIAS HUMANAS

Diretora: Sandra Margarida Nitrini

Vice-Diretor: Modesto Florenzano

DEPARTAMENTO DE LETRAS CLÁSSICAS E VERNÁCULAS

Chefe do Departamento: João Roberto Gomes de Faria

Vice-Chefe do Departamento: Ieda Maria Alves

PROGRAMA DE PÓS-GRADUAÇÃO DE ESTUDOS COMPARADOS DE LITERATURAS DE LINGUA PORTUGUESA

Coordenador: José Nicolau Gregorin Filho

Vice-Coordenador: Helder Garmes

Via Atlântica/Departamento de Letras Clássicas e Vernáculas. Faculdade de Filosofia, Letras e Ciências Humanas. Universidade de São Paulo - n. 21 (2012) -.- São Paulo : Departamento de Letras Clássicas e Vernáculas, 2012.

ISSN 1516-5159

1. Língua Portuguesa 2. Literaturas de expressão portuguesa 3. Literatura comparada I. Universidade de São Paulo. Faculdade de Filosofia, Letras e Ciências Humanas. Departamento de Letras Clássicas e Vernáculas.

CDD-469 


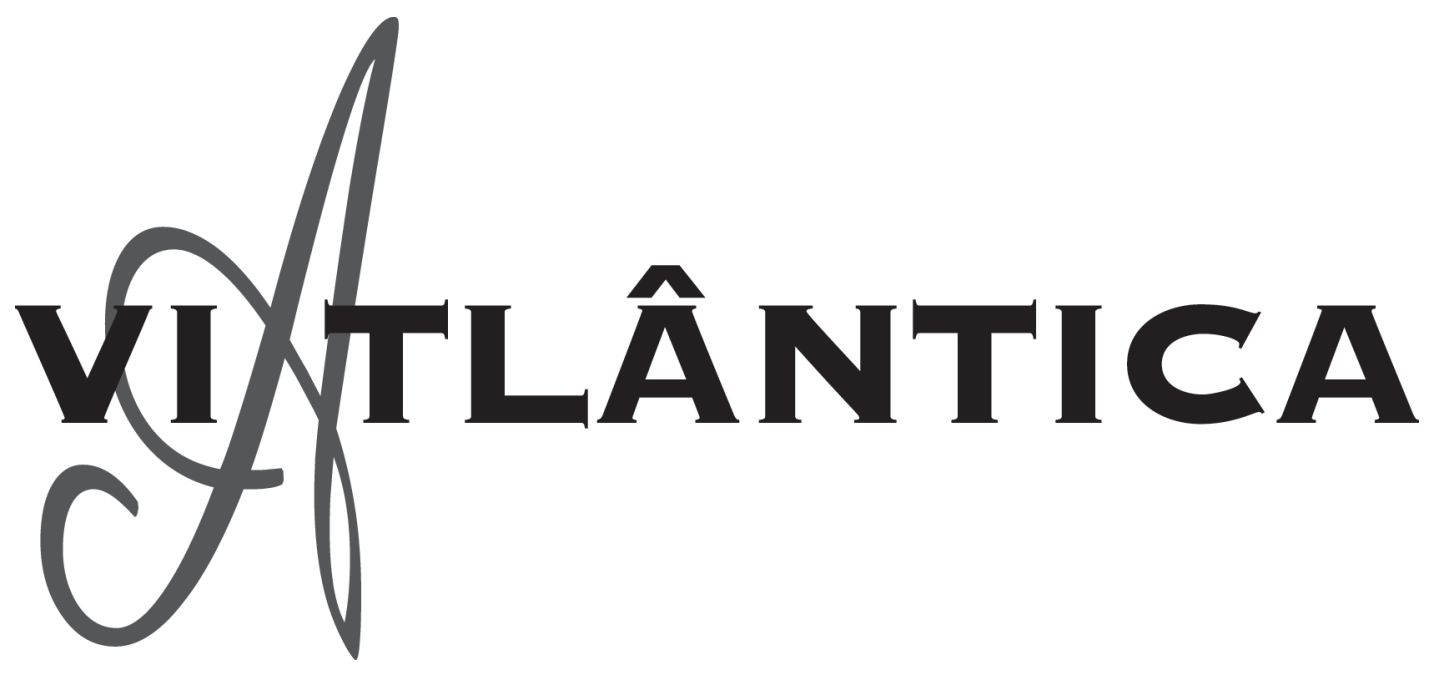

Publicação da Área de Estudos Comparados de Literaturas de Língua Portuguesa Departamento de Letras Clássicas e Vernáculas

Faculdade de Filosofia, Letras e Ciências Humanas Universidade de São Paulo

n. 21 São Paulo 2012 
ORGANIZADORES DESTE NÚMERO

Rita Chaves (Universidade de São Paulo, São Paulo, São Paulo, Brasil)

Tania Macêdo (Universidade de São Paulo, São Paulo, São Paulo, Brasil)

José Luís Cabaço (Universidade Técnica de Moçambique, Maputo, Moçambique)

\title{
COMISSÃO EXECUTIVA
}

Benjamin Abdala Júnior (Universidade de São Paulo, São Paulo, São Paulo, Brasil)

Hélder Garmes (Universidade de São Paulo, São Paulo, São Paulo, Brasil)

Maurício Sales Vasconcelos (Universidade de São Paulo, São Paulo, São Paulo, Brasil)

Rita Chaves (Universidade de São Paulo, São Paulo, São Paulo, Brasil)

Salete de Almeida Cara (Universidade de São Paulo, São Paulo, São Paulo, Brasil)

\section{CONSELHO EDITORIAL}

Ana Pizarro (Universidade de Santiago, Santiago, Santiago, Chile)

Angela Balça (Universidade de Évora, Évora, Alentejo, Portugal)

Benjamin Abdala Júnior (Universidade de São Paulo, São Paulo, São Paulo, Brasil)

Carmen Lúcia Tindó Secco (Universidade Federal do Rio de Janeiro, Rio de Janeiro, Rio de Janeiro, Brasil)

Elza Miné (Universidade de São Paulo, São Paulo, São Paulo, Brasil)

Eneida Leal Cunha (Pontifícia Universidade Católica, Rio de Janeiro, Rio de Janeiro, Brasil)

Francisco Noa (Universidade Eduardo Mondlane, Maputo, Maputo, Moçambique)

João Luís Ceccantini (Universidade Estadual Paulista, Assis, São Paulo, Brasil)

Laura Padilha (Universidade Federal Fluminense, Niterói, rio de Janeiro, Brasil)

Maria Lúcia Dal Farra (Universidade Federal de Sergipe, Aracaju, Sergipe, Brasil)

Maria Luiza Scher Pereira (Universidade Federal de Juiz de Fora, Juiz de Fora, Minas Gerais, Brasil)

Maria Zilda Cunha (Universidade de São Paulo, São Paulo, São Paulo, Brasil)

Nuno Júdice (Universidade Nova de Lisboa, Lisboa, Lisboa, Portugal)

Maria Nazareth Soares (Pontifícia Universidade Católica, Belo Horizonte, Minas Gerais, Brasil)

Regina Zilberman (Universidade Federal do Rio Grande do Sul, Porto Alegre, Rio Grande do Sul, Brasil)

Rejane Vecchia Rocha e Silva (Universidade de São Paulo, São Paulo, São Paulo, Brasil)

Rita Goddet (Universidade de Rennes, Rennes, Bretanha, França)

Roberto Vecchi (Universidade de Bolonha, Bolonha, Emilia-Romanha, Itália)

Sérgio Medeiros (Universidade Federal de Santa Catarina, Florianópolis, Santa Catarina, Brasil)

Walnice Nogueira Galvão (Universidade de São Paulo, São Paulo, São Paulo, Brasil)

\author{
Revisão de Textos Debora Leite David \\ Assessoria Creusa Ribeiro de Lima \\ Marildes Moreira da Silva \\ Editoração Eletrônica RW3 Design \\ Capa e Projeto Gráfico Moema Cavalcanti \\ Impressão e Acabamento Linear B
}

Endereço para correspondência:

Universidade de São Paulo

Faculdade de Filosofia, Letras e Ciências Humanas

Centro de Estudos das Literaturas e Culturas de Língua Portuguesa

Av. Prof. Luciano Gualberto, 403 - sala 101 - CEP 05508-900 - São Paulo - SP - Brasil

Fone: (11) 3091-3751 | e-mail: viatlan@usp.br | celp@usp.br

Via Atlântica, n. 21, 2012

Esta publicação conta com auxílio financeiro da CAPES 


\section{Sumário}

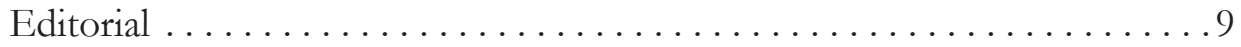

\section{DOSSIÊ: HISTÓRIA, CULTURA E REVOLUÇÃO}

A crise estrutural no sistema-mundo: para onde vamos a partir daqui? . . .15 The structural crisis in the world-system: where do we go from here?

Immanuel Wallerstein

O legado de Amilcar Cabral face aos desafios da ética contemporânea . . . 27 The legacy of Amilcar Cabral before the challenges of contemporary ethics Carlos Lopes

Aquino de Bragança: o intelectual e a independência de África . . . . . . . 45 Aquino de Bragança: the intellectual and the independence of Africa Annamaria Gentili

Discurso revolucionário moçambicano e a escrita do homem novo. .... . 59 Mozambican revolutionary discourse and writing of the "homem novo" Bethania Mariani

"Quem é escrito?" revolução, alteridade, experiências de reescrita e história conectada no contexto da guerra colonial e de libertação em Moçambique . ................................ 75

"Who is written?" Revolution, otherness, experiences rewritten and history connected in the context of colonial war and liberation in Mozambique Maria Benedita Basto 
O fascínio do corpo na obra de Teixeira Gomes ...............93 The fascination of the body in Teixeira Gomes' work Nuno Júdice

Raul Brandão e a tentação histórica: para uma leitura de El-Rei Junot. . . . 101 Raul Brandao and historical temptation: for a reading of El-Rei Junot Otávio Rios Portela

As humanidades: entre a permanência e a finitude ou entre desassossegos e desafios

Humanities: between continuity and finitude or between unrest and challenges Francisco Noa

\section{ENTREVISTA}

Ruy Guerra: cidadão de várias pátrias, passageiro de diferentes revoluções . . . . . . . . . . . . . . . . . . . 133

Ruy Guerra: citizen of many homelands, passenger of different revolutions Rita Chaves e José Luis Cabaço

\section{DEPOIMENTOS}

A insustentável leveza da revolução . . . . . . . . . . . . . . . 147 The unbearable lightness of revolution João Melo

Cultura e revolução - dois nomes, um só verbo? 151

Culture and revolution - two names, just one verb? Mia Couto 


\section{OUTROS TEXTOS}

Ficção e etnografia: o problema da representação em Os papéis do inglês, de Ruy Duarte de Carvalho, e Nove noites, de Bernardo Carvalho . . . . 155 Fiction and ethnography: the problem of representation in Os papéis do inglês, by Ruy Duarte de Carvalho, and Nove noites, by Bernardo Carvalho Anita Martins Rodrigues de Moraes

Ressignificações políticas do espaço mineiro em

Murilo Mendes e Guinard . . . . . . . . . . . . . . . . . . . . . 173

Politics re-signification of Minas Gerais' space in

Murilo Mendes and Guinard

Lucas Mendes Ferreira e Terezinha Maria Scher Pereira

A imagem saturada de agoras

The saturated image of nows

Vera Maquêa

\section{RESENHAS}

Os fantasmas da revolução em Crónica da Rua 513.2, de

João Paulo Borges Coelho. . . . . . . . . . . . . . . . . . . . 201

The ghosts of the revolution in Crónica da Rua 513.2, by

João Paulo Borges Coelho

Nazir Can

Literatura e afrodescendência no Brasil: verdadeiro

com a época e com as palavras. ...................... 207

Literature and afrodescendency in Brazil: true with the

season and with the words

Nonato Gurgel

As malhas dos impérios e alguns trânsitos anticoloniais ...........213 The meshes of the empires and some anti-colonial transits

Rita Chaves 


\section{Editorial}

Um período de crise sistêmica, como o que assola o mundo contemporâneo, convoca reflexões sobre experiências vividas, sobre a relação entre indivíduo e conjuntura, sobre como o fenômeno da mudança estrutural se desdobra nos valores sociais, nos princípios éticos e em suas manifestações estéticas e artísticas, sobre o papel da cultura nos processos de transformação. Em perspectivas interdisciplinares, tais aspectos são abordados no dossiê História, Cultura e Revolução que compõe o número 21 de Via Atlântica.

"A crise estrutural no sistema-mundo: para onde vamos a partir daqui?", de Immanuel Wallerstein abre a discussão. O autor, ex-diretor do Centro de Estudos Fernand Braudel, e um dos mais conceituados teóricos da análise global do sistema capitalista, debruça-se sobre a presente crise estrutural e sobre as opções que se apresentam para a sua superação, contrapondo posições que ele associa ao "espírito de Davos" e ao "espírito de Porto Alegre".

Num mundo conturbado pela crise de valores, também faz sentido rever o pensamento de dois grandes intelectuais e revolucionários que apostaram na ética como base para a transformação que a independência dos países africanos prometia. Dessa forma, especialistas dos estudos africanos foram convidados a apresentar exemplos de intelectuais que souberam articular pensamento e práxis nas suas vivências revolucionárias. Prof. Dr. Carlos Lopes, atualmente Secretário-Executivo da Comissão Econômica para África da Organização das Nações Unidas, traz uma reflexão sobre o pensamento político do guineense Amílcar Cabral, referência incontornável na história da luta contra o colonialismo português, destacando a importância central que, para ele, a questão cultural assumia na libertação e transformação da sociedade colonizada. Sua abordagem dialoga de forma instigante com problemáticas contemporâneas como o multiculturalismo e o "fim das ideologias". Aquino de Bragança, outro intelectual profundamente mergulhado na revolução anti- 
colonial da segunda metade do século XX, tem seu perfil traçado pela Profa. Dra. Annamaria Gentili, da Universidade de Bologna, que com ele trabalhou, em Maputo, no Centro de Estudos Africanos da Universidade Eduardo Mondlane, instituição que ele organizou e dirigiu até o fim de sua vida.

Em "O discurso revolucionário moçambicano e a escrita do homem-novo", Bethania Mariani trabalha os conceitos de "homem novo" e "sociedade nova" nos textos elaborados durante o projeto socialista em Moçambique, como uma tentativa de ruptura político-cultural com o legado português e como enunciado de um projeto ideológico de criação de uma ordem social alternativa.

Ainda sobre a análise do texto em situação de guerra e transformação sociopolítica, Maria-Benedita Basto, tomando um poema de um guerrilheiro da FRELIMO e as letras de canções de militares portugueses, evidencia a tensão entre as narrativas e modelos do Estado que se confrontam e as apropriações dos sujeitos que subvertem a ordem estabelecida. Trata-se de um processo político onde se cruzam três dimensões: a antropológica, a socioafetiva e as dinâmicas performativas de apropriação.

A literatura portuguesa foi visitada nas obras de dois autores. Nuno Júdice convida-nos a uma releitura de Teixeira Gomes, escritor que surge na passagem do século XIX para o século XX, numa proposta que o integra na dimensão panteísta e pagã, que teve o seu início com o poeta Guerra Junqueiro e permite compreender sua associação num conjunto maior de escritores que consagram a substituição da mulher madura pelo mito da adolescente perversa como modelo feminino.

A narrativa histórica de Raul Brandão em El-Rei Junot, articulando efabulação e matéria histórica, é estudada por Otávio Rios Portela, o qual destaca como o autor, em sua prosa historiográfica, dá voz às classes proletárias, aproximando-o de uma história que pretende narrar os vencidos em detrimento dos vencedores.

Retomando, em chave diversa, o discurso da crise do sistema-mundo, Francisco Noa percorre os desassossegos e desafios com que, na sociedade contemporânea, se confronta o estudo das humanidades e a correlata crise de valores. Neste contexto, a literatura, onde coexistem o mundo real e o possível, continua abrindo espaços de liberdade e criatividade nos quais a humanidade se pode reencontrar. 
Cidadão de várias pátrias e passageiros de diferentes revoluções, o cineasta Ruy Guerra detém um capital de experiências vividas sobre a relação entre cultura e processos revolucionários, fossem eles políticos ou estéticos. Em torno desse vasto percurso, Via Atlântica o entrevistou trazendo para o dossiê o seu testemunho direto.

O dossiê do presente número encerra com breves depoimentos sobre a relação entre cultura e revolução, assinados por dois escritores africanos, João Melo, de Angola, e Mia Couto, de Moçambique, ambos participantes dos processos de libertação nacional de seus países.

Na seção "Outros Textos", reiterando a opção metodológica do Programa de Estudos Comparados de Literaturas de Língua Portuguesa, de que Via Atlântica é um veículo essencial, três artigos trazem-nos o comparatismo como base analítica. Em "Ficção e etnografia: o problema da representação em Os papéis do inglês, de Ruy Duarte de Carvalho, e Nove noites, de Bernardo Carvalho", Anita Moraes aborda como os dois autores, trabalhando com literatura e antropologia, se colocam perante as fronteiras entre discurso ficcional e não ficcional.

No segundo texto, "Ressignificações políticas do espaço mineiro em Murilo Mendes e Guignard", Lucas Mendes Ferreira e Teresinha Scher Pereira, colocando em cena a escrita e as artes plásticas, analisam as relações políticas e estéticas entre o poeta surrealista e o pintor modernista na contemplação e revisitação poética de Ouro Preto. Vera Maquêa destaca em "A imagem saturada de agoras", o procedimento da pesquisa minimalista na poesia do angolano Arlindo Barbeitos e na narrativa de Dalton Trevisan.

Três resenhas de obras bastante diversas em sua natureza encerram o presente número de Via Atlântica. Nazir Can apresenta-nos uma obra representativa da ficção moçambicana contemporânea em "Os fantasmas da revolução na Crónica da rua 513.2, de João Paulo Borges Coelho". "Verdadeiro com a época e com as palavras" é o título escolhido por Nonato Gurgel para assinalar a relevância de Literatura e afrodescendência no Brasil, uma antologia da produção literária de autoria negra organizada por Eduardo Assis Duarte, cuja publicação é considerada um marco em nosso cenário editorial. Por fim, em "As malhas dos impérios e alguns trânsitos anticoloniais", Rita Chaves discorre sobre Malhas que os impérios tecem. Textos anticoloniais, contextos pós-coloniais, 
que numa primorosa seleção feita pela estudiosa portuguesa Manuela Ribeiro Sanches oferece-nos textos fundamentais acerca das relações impostas pelo colonialismo, um dos temas essenciais quando o assunto é revolução.

Rita de Cássia Natal Chaves Tania Celestino de Macêdo José Luís Cabaço 